\title{
460.
}

\section{NOTE ON MR FROST'S PAPER ON THE DIRECTION OF LINES OF CURVATURE IN THE NEIGHBOURHOOD OF AN UMBILICUS.}

[From the Quarterly Journal of Pure and Applied Mathematics, vol. x. (1870), pp. 111-113.]

I REMARK as follows:

1. In regard to a quadric surface, it is not, I think, correct to say that the generatrices through an umbilic are curves of curvature; notwithstanding that, as shown p. 80 , the normals at every point of such generatrix lie in one plane and consequently intersect. The way in which these generatrices as quasi-curves-of-curvature present themselves is as follows:

The curves of curvature satisfy a certain differential equation, the complete integral of which gives these curves as the intersections of the given quadric surface by the series of confocal surfaces $\frac{x^{2}}{a^{2}+h}+\frac{y^{2}}{b^{2}+h}+\frac{z^{2}}{c^{2}+h}=1, h$ being the constant of integration of the differential equation. The singular solution of the differential equation, or envelope of the curves of curvature determined as above, gives the umbilicar generatrices.

2. In regard to a surface in general, I think it must be considered, not that there pass through the umbilic three distinct curves, but that the umbilicar curve of curvature is a curve having at the umbilic a triple point, or rather a point at which there are in general three distinct directions of the curve. The umbilicar curve of curvature in fact presents itself as the curve belonging to a certain value of the constant of integration $h$; in order that the curve of curvature may pass through a given point on the surface, $h$ must satisfy a certain quadratic equation, that is for a given point of the surface there are two values of $h$, and therefore two curves of 
curvature; but an umbilic is a point for which (as in effect shown, p. 81, for the particular case of a quadric surface) the two values of $h$ become equal; that is, there is through the umbilic only a singular curve of curvature; but $\frac{d y}{d x}$ is determined by a cubic equation, and the umbilic is thus (as just mentioned) a point at which there are in general three distinct directions of the curve.

3. Some researches on the subject are contained in my paper "On Differential Equations and Umbilici," Phil. Mag., vol. xxvi. (1863), pp. $373-379$ and $441-452$, [330]. It is noticeable that in the integral equations which $I$ have there obtained for the differential equations $c y\left(p^{2}-1\right)+(a-c) x p=0$, and the more general form $(b x+c y)\left(p^{2}-1\right)+2(f x+g y)=0$, which belong to the neighbourhood of an umbilic, the curve through the umbilic does break up into three distinct curves; and the same is the case with the umbilic on the surface $x y z=1$ presently referred to.

4. In the paper "Mémoire sur les surfaces orthogonales," Liouv, t. XII. (1847), pp. 241-254, M. Serret has given two very remarkable cases of three systems of surfaces intersecting each other at right angles, and consequently in the curves of curvature of the surfaces of each system. It was only on referring to this paper, in connexion. with that of $\mathrm{Mr}$ Frost, that I perceived an obvious enough simplification of M. Serret's formulæ, whereby it appears that the curves of curvature on the surface $x y z=1$ are given as the intersection of this surface with the series of surfaces

$$
h=\left(x^{2}+\omega y^{2}+\omega^{2} z^{2}\right)^{\frac{3}{2}}+\left(x^{2}+\omega^{2} y^{2}+\omega z^{2}\right)^{\frac{3}{2}},
$$

where $\omega$ is an imaginary cube root of unity; the rationalised equation is of the twelfth order in $(x, y, z)$, and for the particular value $h=0$, reduces itself as is easily seen to $0=\left(y^{2}-z^{2}\right)^{2}\left(z^{2}-x^{2}\right)^{2}\left(x^{2}-y^{2}\right)^{2}$. The point $x=y=z=1$ is obviously an umbilic on the surface $x y z=1$, and the corresponding value of $h$ being $h=0$, the equation just obtained determines the umbilicar curves of curvature, viz. combining therewith the equation $x y z=1$ of the surface, we have the three hyperbolic curves

$$
\left(y=z, x y^{2}=1\right), \quad\left(z=x, y z^{2}=1\right), \quad\left(x=y, z x^{2}=1\right) .
$$

